\title{
FIXED POINT ITERATIONS USING INFINITE MATRICES
}

\author{
BY
}

\section{B. E. RHOADES}

ABSTRACT. Let $E$ be a closed, bounded, convex subset of a Banach space $X, f: E \rightarrow E$. Consider the iteration scheme defined by $\bar{x}_{0}=x_{0} \in E$, $\bar{x}_{n+1}=f\left(x_{n}\right), x_{n}=\sum_{k=0}^{n} a_{n k} \bar{x}_{k}, n \geq 1$, where $A$ is a regular weighted mean matrix. For particular spaces $X$ and functions $f$ we show that this iterative scheme converges to a fixed point of $f$.

Let $X$ be a normed linear space, $E$ a nonempty closed bounded, convex subset of $X, f: E \rightarrow E$ possessing at least one fixed point in $E$, and $A$ an infinite matrix. Given the iteration scheme

$$
\begin{gathered}
\bar{x}_{0}=x_{0} \in E, \\
\bar{x}_{n+1}=f\left(x_{n}\right), \quad n=0,1,2, \ldots, \\
x_{n}=\sum_{k=0}^{n} a_{n k} \bar{x}_{k}, \quad n=1,2,3, \ldots,
\end{gathered}
$$

it is reasonable to ask what restrictions on the matrix $A$ are necessary and/or sufficient to guarantee that the above iteration scheme converges to a fixed point of $f$.

During the past few years several mathematicians have obtained results using iteration schemes of the form (1)-(3) for certain classes of infinite matrices. In this paper we establish generalizations of several of these results as well as point out some of the duplication and overlap of the work of these authors.

An infinite matrix $A$ is called regular if it is limit preserving over $c$, the space of convergent sequences; i.e., if $x \in c, x_{n} \rightarrow l$, then $A_{n}(x)=\Sigma_{k} a_{n k} x_{k} \rightarrow$ l. A matrix is called triangular if it has only zeros above the main diagonal, and a triangle if it is triangular and all of its main diagonal entries are nonzero. We shall confine our attention to regular triangular matrices $A$ satisfying

$$
0 \leq a_{n k} \leq 1, \quad n, k=0,1,2, \cdots
$$

Received by the editors November 21, 1972 and, in revised form, October 1, 1973. AMS (MOS) subject classifications (1970). Primary 26A18, 40G05, 40G99, 47H10.

Key words and phrases. Cesàro matrix, contraction, fixed point iterations, quasinonexpansive, regular matrix, strictly pseudocontractive, weighted mean matrix. 


$$
\sum_{k=0}^{n} a_{n k}=1, \quad n=0,1,2, \ldots .
$$

Conditions (4) and (5) are obviously necessary in order to ensure that $x_{n}$ and $\bar{x}_{n}$ in (2) and (3) remain in $E$. The scheme (1)-(3) is generally referred to as the Mann process, in the light of [13].

Barone [1] observed that a sufficient condition for a regular matrix $A$ to transform each bounded sequence into a sequence whose set of limit points is connected is that $A$ satisfy

$$
\lim _{n} \sum_{k=0}^{\infty}\left|a_{n k}-a_{n-1, k}\right|=0 .
$$

In [18] we posed the following conjecture:

Conjecture. Let $f$ be a continuous mapping of $[a, b]$ into itself, $A$ a regular matrix satisfying (4)-(6). Then the iteration scheme defined by $(1)-(3)$ converges to a fixed point of $f$.

The conjecture need not remain true if condition (6) is removed. To see this, let $A$ be the identity matrix, $[a, b]=[0,1], f(x)=1-x$, and choose $x_{0}=0$. (Note Remark 3 following Theorem 8.)

The conjecture is true for a large class of weighted mean matrices (see, e.g., $[8$, p. 57] for the definition and basic properties of weighted mean matrices) as we now show.

$A$ weighted mean method is a regular triangular method $A=\left(a_{n k}\right)$ defined by $a_{n k}=p_{k} / P_{n}$, where the sequence $\left\{p_{n}\right\}$ satisfies $p_{0}>0, p_{n} \geq 0$ for $n>0, P_{n}=$ $\Sigma_{k=0}^{n} p_{k}$ and $P_{n} \rightarrow \infty$ as $n \rightarrow \infty$. It is easy to verify that such a matrix satisfies (6) if and only if $p_{n} / P_{n} \rightarrow 0$ as $n \rightarrow \infty$.

Theorem 1. Let $A$ be a regular weighted mean method satisfying (6), $f$ a continuous mapping from $E=[a, b]$ into itself. Then the iteration scbeme (1)(3) converges to a fixed point of $f$.

Proof. There is no loss of generality in assuming $[a, b]=[0,1]$. Any regular weighted mean method automatically satisfies conditions (4) and (5). Using (3) we may write

$$
x_{n+1}=\left(p_{n+1} / P_{n+1}\right)\left(f\left(x_{n}\right)-x_{n}\right)+x_{n} \text {. }
$$

Since $x_{n}, f\left(x_{n}\right) \in[0,1]$, we have, from (7), $\left|x_{n+1}-x_{n}\right| \leq p_{n+1} / P_{n+1} \rightarrow 0$ as $n \rightarrow \infty$.

One now parrots the proof in $[6$, p. 325$]$ to establish that $\left\{x_{n}\right\}$ converges. It remains to show that $\left\{x_{n}\right\}$ tends to a fixed point of $f$. 
Fact. Let $A$ be any regular matrix, $f$ as defined above. If the iteration scheme (1)-(3) converges, it converges to a fixed point of $f$.

Proof. Let $x=\left\{x_{n}\right\}, \bar{x}=\left\{\bar{x}_{n}\right\}, y=\lim _{n} x_{n}$. By the continuity of $f$, $\lim _{n} f\left(x_{n}\right)=f(y)$. Since $\bar{x}_{n+1}=f\left(x_{n}\right)$ for each $n, \lim _{n} \bar{x}_{n}=f(y)$. But $A$ is a regular matrix. Hence $y=\lim _{n} x_{n}=\lim _{n} A_{n}(\bar{x})=f(y)$.

A proof of the Fact also appears in [13], and, for arbitrary linear normed spaces, in [4] and [16]. In [6] a more complicated and summability independent proof is used.

One obtains the theorem of [6] by setting $p_{n} \equiv 1$ in Theorem 1 .

J. Reinermann [16] defines a summability matrix $A$ by

$$
\begin{aligned}
a_{n k} & =c_{k} \prod_{j=k+1}^{n}\left(1-c_{j}\right), & & k<n, \\
& =c_{n}, & & k=n, \\
& =0, & & k>n,
\end{aligned}
$$

where the real sequence $\left\{c_{n}\right\}$ satisfies (i) $c_{0}=1$, (ii) $0<c_{n}<1$ for $n \geq 1$, and (iii) $\Sigma_{k} c_{k}$ diverges. (It is easy to verify that $A$ is regular and satisfies conditions (4) and (5). Actually Reinermann permits $c_{n}=1$ in order to take care of the identity matrix, but in all interesting applications the restriction $c_{n}<1$ is imposed.) He then defines the iteration scheme (1) and $x_{n+1}=\sum_{k=0}^{n} a_{n k} f\left(x_{k}\right)$, which can be written in the form

$$
x_{n+1}=\left(1-c_{n}\right) x_{n}+c_{n} f\left(x_{n}\right)
$$

and establishes the following.

Theorem R1 [16, p. 211]. Let $a, b \in R, a<b, E=[a, b], f: E \rightarrow E, f$ continuous and with at most one fixed point. With $A$ as defined in (8) and with $\left\{c_{n}\right\}$ satisfying (i)-(iii) and $\lim _{n} c_{n}=0$, the iteration scheme (1), (9) converges to the fixed point of $f$.

The same iteration scheme has been defined independently by Outlaw and Groetsch [15] and Dotson [4]. In fact, Theorem 2 of [4] is a characterization of the method described by ( 8 ) and (i)-(iii).

With the choice $c_{n}=(n+1)^{-1}$, Theorem R1 reduces to that in [13].

The matrix of (8) with $\left\{c_{n}\right\}$ satisfying (i)-(iii) is a regular weighted mean matrix. For, set $a_{n k}=p_{k} / P_{n}^{n}, k \leq n$. Then $p_{k} / p_{k+1}=a_{n k} / a_{n, k+1}=$ $c_{k}\left(1-c_{k+1}\right) / c_{k+1}$, which can be solved to obtain

$$
p_{k}=c_{k} p_{0} / \prod_{j=1}^{k}\left(1-c_{j}\right), \quad k>0 .
$$


By induction one can show that $P_{n}=p_{0} / \Pi_{j=1}^{n}\left(1-c_{j}\right)$ for $n>0$. Since $\Sigma_{k} c_{k}$ diverges, the product must diverge to 0 . Therefore $P_{n} \rightarrow \infty$ as $n \rightarrow \infty$ and the weighted mean method $\left(\bar{N}, p_{n}\right)$ with $p_{n}$ defined by $(10)$ is regular. Also, each $p_{k}>0$.

Conversely, let $\left(\bar{N}, p_{n}\right)$ be a regular weighted mean method with each $p_{k}>0$ and define $\left\{c_{n}\right\}$ by

$$
c_{n}=p_{n} / P_{n}, \quad n \geq 0
$$

Then $c_{0}=1$, and, since each $p_{k}>0,0<c_{n}<1$ for all $n>0$. Now from (11), $1-c_{n}=P_{n-1} / P_{n}$, which leads to $P_{n}=p_{0} / \Pi_{j=1}^{n}\left(1-c_{j}\right)$. Therefore $p_{k} / P_{n}=$ $c_{k} \Pi_{j=k+1}^{n}\left(1-c_{j}\right)$ and $A$ has the form (8). Moreover, $\Sigma_{k} c_{k}$ diverges because $P_{n} \rightarrow$ $\infty$ as $n \rightarrow \infty$. Since $c_{n}=p_{n} / P_{n}$, the condition $\lim _{n} c_{n}=0$ is the same as $\left(\bar{N}, p_{n}\right)$ satisfying (6).

We point out, however, that even though the matrices involved are the same, the iteration schemes (1)-(3) and (1), (9) are different. Scheme (1)-(3) takes the form $x=A z$, where $z=\left\{x_{0}, f\left(x_{0}\right), f\left(x_{1}\right), \cdots\right\}$; whereas (1) and (9) become $x=A w$, where $w=\left\{f\left(x_{0}\right), f\left(x_{1}\right), \cdots\right\}$. In other words the first scheme uses a translate of $w$. However, since $f$ is continuous, it is easy to verify, using the Fact, that each method converges to the same fixed point.

Therefore Theorems 1 and $\mathrm{R} 1$ are equivalent statements when $f$ has exactly one fixed point and $\left\{c_{n}\right\}$ satisfies (i)-(iii). Theorem 1 generalizes Theorem R1 to those cases where $/$ may have more than one fixed point.

Because the iteration scheme defined by (1), (9) is notationally simpler, the remaining theorems of this paper will be stated and proved in terms of a matrix $A$ defined by (8) with $\left\{c_{n}\right\}$ satisfying (i)-(iii). The reader should be aware, however, that each of these the orems has an equivalent theorem stated in terms of a regular weighted mean matrix $\left(\bar{N}, p_{n}\right)$ and a corresponding condition on $\left\{p_{n} / P_{n}\right\}$. The conclusions remain unchanged even though the $f$ involved may not be continuous.

Hillam [9] has shown the conjecture to be false and has established the following result, which is a slight generalization of The orem 1 .

Theorem Hl $[9$, p. 16]. Let $f:[0,1] \rightarrow[0,1], f$ continuous, $A$ a regular triangular matrix satisfying (4)-(6) and

$$
\sum_{k=0}^{n}\left|a_{n+1, k}-\left(1-a_{n+1, k+1}\right) a_{n k}\right|=o\left(a_{n+1, n+1}\right) .
$$

If, in addition, 


$$
\sum_{n=1}^{\infty} \sum_{k=0}^{n}\left|a_{n+1, k}-\left(1-a_{n+1, k+1}\right) a_{n k}\right|<\infty,
$$

then the iteration scheme (1)-(3) converges to a fixed point of $f$.

For an arbitrary Banach space $X$, continuity is not an adequate restriction to guarantee convergence to a fixed point. Fixed point the orems have been established for functions satisfying a variety of growth conditions. We shall focus on five of these. Using the notation of [2] we shall call $P_{1}=\{f \mid f$ is contractive on $E$; i.e., $\|f(x)-f(y)\| \leq\|x-y\|$ for all $x, y \in X\}$, and $P_{2}=\{f \| f$ is strictly pseudocontractive on $X$; i.e., there exists a positive constant $k<1$ such that $\|f(x)-f(y)\|^{2} \leq\|x-y\|^{2}+k\|(I-f) x-(I-f) y\|^{2}$ for all $\left.x, y \in X\right\}$.

In [19] a number of definitions of contractive-type mappings are compared. We shall consider two of the more general ones.

Let $\alpha, \beta, \gamma$ be real nonnegative numbers satisfying $\alpha<1, \beta, \gamma<1 / 2$. We shall say that $f: X \rightarrow X$ satisfies condition $Z$ if, for each pair of points $x, y \in X$, at least one of the following conditions is satisfied:

(i) $\|f(x)-f(y)\| \leq a\|x-y\|$,

(ii) $\|f(x)-f(y)\| \leq \beta[\|x-f(x)\|+\|y-f(y)\|]$, or

(iii) $\|f(x)-f(y)\| \leq \gamma[\|x-f(y)\|+\|y-f(x)\|]$.

Suppose, for each $x, y \in X$, there exist nonnegative numbers $q(x, y), r(x, y)$, $s(x, y)$, and $t(x, y)$ such that

$$
\sup _{x, y \in X}\{q(x, y)+r(x, y)+s(x, y)+2 t(x, y)\}=\lambda<1
$$

Let

$$
\begin{aligned}
C=\{f \mid\|f(x)-f(y)\| \leq & q(x, y)\|x-y\|+r(x, y)\|x-f(x)\| \\
& +s(x, y)\|y-f(y)\|+t(x, y)[\|x-f(y)\|+\|y-f(x)\|]\} .
\end{aligned}
$$

Let $Q=\{f \mid f$ is quasi-nonexpansive on $X$; i.e., for each fixed point $p$ of $f$ in $X$, $\|f(x)-f(p)\| \leq\|x-p\|\}$.

Clearly $P_{1} \subseteq P_{2}$ and, if $f$ has a fixed point in $X, P_{1} \subseteq Q$, but $P_{2}$ and $Q$ are independent. Using the example in [4], let $X$ be the reals, $f$ defined by $f(x)=$ $(x / 2) \sin (1 / x), 0<x \leq 1, f(0)=0$. Then 0 is a fixed point of $f$, and it can be shown that $f \in Q$. To show that $f \notin P_{2}$, pick $x=2 /(4 n+1) \pi, y=2 /(4 n+3) \pi$, $n>1$. Now consider a function $f$ defined by $f(x)=(-x / a)+1,0 \leq x \leq a, 1 / 3<$ $a<1 / 2$, and $f(x)=0$ for $a<x \leq 1$. Then $f \in P_{2}$ with $k=(1-a) /(1+a)$, but $f \notin Q$. In [19] it is shown that $Z$ is a proper subset of $C$. From [21], if $f \in Z$ then $f$ has a fixed point in $X$. It is easy to show that $Z \subseteq Q$.

Definitions $C$ and $P_{2}$ are independent. The $f$ in Example 1 of [3] is not continuous, hence $f \notin P_{2}$. The $f$, in the above example, which belongs to $P_{2}$ 
and not to $Q$, also does not belong to $C$.

Sets $C$ and $Q$ are independent. Define $f$ by $f(x)=0,0 \leq x \leq 1 / 2, f(x)=1 / 2$. $1 / 2<x \leq 1$. Then $f \in Q$, but $f \notin C$. On the other hand, if $f(x)=1 / 4,0 \leq x \leq 1 / 3$, $f(x)=0,1 / 3<x \leq 1$, then $f \in C$ and $f \notin Q$.

Sets $Z$ and $\bar{P}_{2}$ are independent. Since $Z \subseteq C$, there exists an $f \in P_{2}, f \notin Z$. Example 1 of [3] also satisfies $Z$, but not $P_{2}$.

The most general theorem for a quasi-nonexpansive mapping on a strictly convex Banach space is the following.

Theorem 2 [4, Theorem 3]. Let $X$ be a strictly convex Banach space, $E$ a closed convex subset of $X, f: X \rightarrow X, f$ continuous, $f \in Q$ on $X$ and $f(E) \subset K \subset X$ for some compact set $K$. Let $A$ be defined by (8) with $\left\{c_{n}\right\}$ satisfying (i)-(iii) and such that $\left\{c_{n}\right\}$ bas a cluster point in $(0,1)$. Then the iteration scbeme (1), (9) converges strongly to a fixed point of $f$.

For uniformly convex spaces we combine Theorem 5 of [4] with Corollary 1 and comment (ii) from [7].

Theorem 3. Let $X$ be a uniformly convex Banach space, $f: E \rightarrow E, E$ a closed convex subset of $X, f \in Q$ with at least one fixed point in $E$, and $I-f$ closed. Let $A$ be defined by (8) with $\left\{c_{n}\right\}$ satisfying (i), (ii), and (iv): $\Sigma_{k} c_{k}\left(1-c_{k}\right)$ diverges. If, in (9), $\left\{x_{n}\right\}$ clusters strongly at some $p \in E$, then $f(p)=p$, and $\left\{x_{n}\right\}$ and $\left\{\bar{x}_{n}\right\}$ (defined by (2)) converge to $p$.

The following example shows that continuity is a necessary condition in Theorem 2, and that $I-f$ closed is a necessary condition in Theorem 3. Let $X$ be the reals, $E=[0,1], f$ defined by $f(x)=1 / 2,0 \leq x \leq 1 / 2, f(x)=1,1 / 2<x<1$, $f(1)=3 / 4$, and $A$ the matrix defined by (8) with $c_{n} \equiv \alpha$ for $n \geq 1$, for some $0<a<1$. $f \in Q$ and has $1 / 2$ as its only fixed point. Choose $x_{0}=1$. One can show that $3 / 4<x_{n}<1$ and $x_{n}$ in $n$ for all $n \geq 2$.

Since condition (iv) on $\left\{c_{n}\right\}$ implies (iii), the theorem of [7] shows that, in Lemma 2 of [16], one can replace the conditions: $\left\{c_{n}\right\} \downarrow$ in $n$ and the convexity moduli $\delta_{n}$ chosen so that $\Sigma_{n} \delta_{n}$ diverges, by (iv) and still retain the conclusion $\lim _{n}\left\|f\left(x_{n}\right)-x_{n}\right\|=0$. The orem 3 is therefore a generalization of Theorem 3 of [16], with the hypothesis $I-f$ closed replaced by $f^{n_{0}}$ completely continuous for some $n_{0}$. Theorem 1 of [14] and Theorem 5 of [4] are special cases of Theorem 3.

By restricting the class of functions to $Z$, a stronger version of Theorem 3 can be proved.

Theorem 4. Let $X$ be a uniformly convex Banach space, $E$ a closed convex 
subset of $X, f: E \rightarrow E, f \in Z$. Let $A$ be defined by (8) with $\left\{c_{n}\right\}$ satisfying (i), (ii), and (iv). Then $\left\{x_{n}\right\}$ of (1), (9) converges to the fixed point of $f$.

Proof. Theorem 1 of [21] shows that $f$ has a unique fixed point in $X$. Call it $p$. For any $x \in E$,

$$
\left\|x_{n+1}-p\right\| \leq\left(1-c_{n}\right)\left\|x_{n}-p\right\|+c_{n}\left\|f\left(x_{n}\right)-p\right\| .
$$

Since $Z \subseteq Q,\left\|f\left(x_{n}\right)-p\right\| \leq\left\|x_{n}-p\right\|$. Thus $\left\{\left\|x_{n}-p\right\|\right\} \downarrow$ in $n$. Also, $\left\|x_{n}-f\left(x_{n}\right)\right\|=$ $\left\|\left(x_{n}-p\right)-\left(f\left(x_{n}\right)-p\right)\right\| \leq 2\left\|x_{n}-p\right\|$. We shall assume that there is a number $a>0$ such that $\left\|x_{n}-p\right\| \geq a$ for all $n$.

Assume $\left\{\left\|x_{n}-f\left(x_{n}\right)\right\|\right\}$ does not converge to zero. Then there are two possibilities. Either there exists an $\epsilon>0$ such that $\left\|x_{n}-f\left(x_{n}\right)\right\| \geq \epsilon$ for all $n$ or $\lim _{n}\left\|x_{n}-f\left(x_{n}\right)\right\|=0$. In the first case, use the lemma of [7] with $b=2 \delta\left(\epsilon /\left\|x_{0}-p\right\|\right)$ to get

$$
\begin{aligned}
\left\|x_{n+1}-p\right\| & \leq\left(1-c_{n}\left(1-c_{n}\right) b\right)\left\|\left(x_{n}-p\right)\right\| \\
& \leq\left\|x_{n-1}-p\right\|-c_{n-1}\left(1-c_{n-1}\right) b\left\|x_{n / 1}-p\right\|-\left\|x_{n}-p\right\| b c_{n}\left(1-c_{n}\right) \\
& \leq\left\|x_{n-1}-p\right\|-\left\|x_{n}-p\right\| b\left(c_{n-1}\left(1-c_{n-1}\right)+c_{n}\left(1-c_{n}\right)\right) .
\end{aligned}
$$
fore,

By induction, $a \leq\left\|x_{n+1}-p\right\| \leq\left\|x_{0}-p\right\|-\left\|x_{n}-p\right\| b \Sigma_{k=0}^{n} c_{k}\left(1-c_{k}\right)$. There-

$$
a\left(1+b \sum_{k=0}^{n} c_{k}\left(1-c_{k}\right)\right) \leq\left\|x_{0}-p\right\|,
$$

a contradiction since $\left\{c_{n}\right\}$ satisfies (iv).

In the second case, there exists a subsequence such that $\lim _{k}\left\|x_{n_{k}}-f\left(x_{n_{k}}\right)\right\|=0$.

If $x_{n_{k}}, x_{n_{l}}$ satisfy (i) of $Z$, then

$$
\begin{aligned}
\left\|f\left(x_{n_{k}}\right)-f\left(x_{n_{l}}\right)\right\| & \leq a\left\|x_{n_{k}}-x_{n_{l}}\right\| \\
& \leq a\left[\left\|x_{n_{k}}-f\left(x_{n_{k}}\right)\right\|+\left\|f\left(x_{n_{k}}\right)-f\left(x_{n_{l}}\right)\right\|+\left\|f\left(x_{n_{l}}\right)-x_{n_{l}}\right\|\right],
\end{aligned}
$$

$\left\|f\left(x_{n_{k}}\right)-f\left(x_{n_{l}}\right)\right\| \leq a(1-a)^{-1}\left[\left\|x_{n_{k}}-f\left(x_{n_{k}}\right)\right\|+\left\|f\left(x_{n_{l}}\right)-x_{n_{l}}\right\|\right]$.

If $x_{n_{k}}, x_{n_{l}}$ satisfy (ii) of $Z$, then

$$
\left\|f\left(x_{n_{k}}\right)-f\left(x_{n_{l}}\right)\right\| \leq \beta\left[\left\|x_{n_{k}}-f\left(x_{n_{k}}\right)\right\|+\left\|x_{n_{l}}-f\left(x_{n_{l}}\right)\right\|\right] .
$$

If $x_{n_{k}}, x_{n_{l}}$ satisfy (iii) of $Z$, then 
so that

$$
\left\|f\left(x_{n_{k}}\right)-f\left(x_{n_{l}}\right)\right\| \leq \gamma\left[\left\|x_{n_{k}}-f\left(x_{n_{l}}\right)\right\|+\left\|x_{n_{l}}-f\left(x_{n_{k}}\right)\right\|\right]
$$

$$
\left\|f\left(x_{n_{k}}\right)-f\left(x_{n_{l}}\right)\right\| \leq \gamma(1-2 \gamma)^{-1}\left[\left\|x_{n_{k}}-f\left(x_{n_{k}}\right)\right\|+\left\|x_{n_{l}}-f\left(x_{n_{l}}\right)\right\|\right] .
$$

Therefore $\left\{f\left(x_{n_{k}}\right)\right\}$ is Cauchy, hence convergent. Call the limit $u$. Then $\lim _{k} x_{n_{k}}=\lim _{k} f\left(x_{n_{k}}\right)=u$.

For each $k$,

$$
\|u-f(u)\| \leq\left\|u-x_{n_{k}}\right\|+\left\|x_{n_{k}}-f\left(x_{n_{k}}\right)\right\|+\left\|f\left(x_{n_{k}}\right)-f(u)\right\| .
$$

If $x_{n_{k}}, u$ satisfy (i), then $\left\|f\left(x_{n_{k}}\right)-f(u)\right\|<\left\|x_{n_{k}}-u\right\|$. If $x_{n_{k}}, u$ satisfy (ii), then $\left\|f\left(x_{n_{k}}\right)-f(u)\right\| \leq \beta\left[\left\|x_{n_{k}}-f\left(x_{n_{k}}\right)\right\|+\|u-f(u)\|\right]$, so that

$$
\|u-f(u)\| \leq\left[\left\|u-x_{n_{k}}\right\|+(1+\beta)\left\|x_{n_{k}}-f\left(x_{n_{k}}\right)\right\|\right] /(1-\beta) .
$$

If $x_{n_{k}}, u$ satisfy (iii), then

$$
\begin{aligned}
\left\|f\left(x_{n_{k}}\right)-f(u)\right\| & \leq \gamma\left[\left\|x_{n_{k}}-f(u)\right\|+\left\|u-f\left(x_{n_{k}}\right)\right\|\right] \\
& \leq \gamma\left[\left\|x_{n_{k}}-f\left(x_{n_{k}}\right)\right\|+\left\|f\left(x_{n_{k}}\right)-f(u)\right\|+\left\|u-f\left(x_{n_{k}}\right)\right\|,\right.
\end{aligned}
$$

or

$$
\left\|f\left(x_{n_{k}}\right)-f(u)\right\| \leq \gamma(1-\gamma)^{-1}\left[\left\|x_{n_{k}}-f\left(x_{n_{k}}\right)\right\|+\left\|u-f\left(x_{n_{k}}\right)\right\|\right]
$$

Hence $u=f(u)$.

Since $p$ is the unique fixed point of $f, p=u$. The two conditions $\lim _{k} x_{n_{k}}=$ $u=p$ and $\left\{\left\|x_{n}-p\right\|\right\} \downarrow$ in $n$ yield $\lim _{n} x_{n}=p$.

It has been shown in [19] that the contractive condition of Kannan [11] is a special case of that of [20]. Thus Theorems 2 and 3 of [11] are special cases of Theorem 4 with $c_{n} \equiv 1 / 2$. Theorem 3 of [12] is the special case of Theorem 4 with $c_{n} \equiv \lambda, 0<\lambda<1$.

Theorem 5. Let $X$ be a Banacb space, $f: X \rightarrow X, f \in C$. Let $A$ be defined by (8) with $\left\{c_{n}\right\}$ satisfying (i), (ii) and bounded away from zero. Then, if $\left\{x_{n}\right\}$, defined by (1), (9) converges to a point $p, p$ is the unique fixed point of $f$ in $X$.

Proof. For each $n, x_{n+1}-x_{n}=c_{n}\left(f\left(x_{n}\right)-x_{n}\right)$. Since $\lim _{n} x_{n}=p$, $\lim _{n}\left\|x_{n+1}-x_{n}\right\|=0$. Since $\left\{c_{n}\right\}$ is bounded away from zero, $\lim _{n}\left\|f\left(x_{n}\right)-x_{n}\right\|=0$. 
For $f \in C$, writing $q$ for $q\left(x_{n}, p\right)$, and similarly for $r, s$, and $t$,

$$
\begin{aligned}
\left\|f\left(x_{n}\right)-f(p)\right\| \leq & q\left\|x_{n}-p\right\|+r\left\|x_{n}-f\left(x_{n}\right)\right\| \\
& +s\|p-f(p)\|+t\left[\left\|x_{n}-f(p)\right\|+\left\|p-f\left(x_{n}\right)\right\|\right], \\
\|p-f(p)\| \leq & \left\|p-x_{n}\right\|+\left\|x_{n}-f\left(x_{n}\right)\right\|+\left\|f\left(x_{n}\right)-f(p)\right\|, \\
\left\|x_{n}-f(p)\right\| \leq & \left\|x_{n}-f\left(x_{n}\right)\right\|+\left\|f\left(x_{n}\right)-f(p)\right\|,
\end{aligned}
$$

and

$$
\left\|p-f\left(x_{n}\right)\right\| \leq\left\|p-x_{n}\right\|+\left\|x_{n}-f\left(x_{n}\right)\right\|
$$

Therefore

$$
\begin{aligned}
\left\|f\left(x_{n}\right)-f(p)\right\| & \leq\left[(q+s+t)\left\|x_{n}-p\right\|+(r+s+2 t)\left\|x_{n}-f\left(x_{n}\right)\right\|\right] /(1-s-t) \\
& \leq \lambda(1-\lambda)^{-1}\left[\left\|x_{n}-p\right\|+\left\|x_{n}-f\left(x_{n}\right)\right\|\right] ;
\end{aligned}
$$

i.e., $\lim _{n} f\left(x_{n}\right)=f(p)$. $\|f(p)-p\| \leq\left\|f(p)-f\left(x_{n}\right)\right\|+\left\|f\left(x_{n}\right)-x_{n}\right\|+\left\|x_{n}-p\right\|$. Hence $p=f(p)$. From $[3], p$ is unique.

Since $\left\{c_{n}\right\}$ is bounded away from zero, $\Sigma_{c_{k}}$ diverges, and $A$ is a regular method equivalent to convergence. From the viewpoint of summability, Theorem 5 is a natural result. A special case of Theorem 5 appears in [11]. The following result is the analogous one for functions in $P_{2}$.

Theorem 6. Let $X$ be a Banach space, $f: X \rightarrow X, f \in P_{2}$. Let $A$ be defined by (8) with $\left\{c_{n}\right\}$ satisfying (i), (ii) and bounded away from zero. Then, if $\left\{x_{n}\right\}$ defined by (1), (9) converges to a point $p, p$ is a fixed point of $f$.

Proof. Since $f \in P_{2},\left\|f\left(x_{n}\right)-f(p)\right\| \leq(1+\sqrt{ } k)\left\|x_{n}-p\right\| /(1-\sqrt{ } k)$, so that $\lim _{n} f\left(x_{n}\right)=f(p)$. As in the proof of Theorem 5, $\lim _{n}\left\|f\left(x_{n}\right)-x_{n}\right\|=0$. Thus

$$
\|f(p)-p\| \leq\left\|f(p)-f\left(x_{n}\right)\right\|+\left\|f\left(x_{n}\right)-x_{n}\right\|+\left\|x_{n}-p\right\| \text { and } f(p)=p \text {. }
$$

We now establish some results when $X$ is a Hilbert space and $f \in C, P_{2}$, or Q.

Theorem 7. Let $H$ be a Hilbert space, $f \in C, f: E \rightarrow E, E$ a closed convex subset of $H$. Let $A$ be defined by (8) with $\left\{c_{n}\right\}$ satisfying (i)-(iii) and $\overline{\lim } c_{n}<$ $1-\lambda^{2}$. Then the iteration scheme (1), (9) converges to the fixed point of $f$.

Proof. From [3] $f$ has a unique fixed point $p \in H$. Let $u, v \in H, \alpha, \beta$ any nonnegative real numbers with $\alpha+\beta=1$. Then one can expand and add the inner products $(\alpha u+\beta v, \alpha u+\beta v)$ and $(\alpha(u-v), \beta(u-v))$ to obtain the identity

$$
\|\alpha u+\beta v\|^{2}=\alpha\|u\|^{2}+\beta\|v\|^{2}-\alpha \beta\|u-v\|^{2} \text {. }
$$


Using this identity and (9), we have, for each positive integer $n$,

(12) $\left\|x_{n+1}-p\right\|^{2}=\left(1-c_{n}\right)\left\|x_{n}-p\right\|^{2}+c_{n}\left\|f\left(x_{n}\right)-p\right\|^{2}-c_{n}\left(1-c_{n}\right)\left\|f\left(x_{n}\right)-x_{n}\right\|^{2}$.

Since $f \in C$,

$$
\left\|f\left(x_{n}\right)-p\right\| \leq q\left\|x_{n}-p\right\|+r\left\|x_{n}-f\left(x_{n}\right)\right\|+t\left[\left\|x_{n}-f(p)\right\|+\left\|p-f\left(x_{n}\right)\right\|\right],
$$

i.e.,

$$
\begin{aligned}
\left\|f\left(x_{n}\right)-p\right\| & \leq\left[(q+t)\left\|x_{n}-p\right\|+r\left\|x_{n}-f\left(x_{n}\right)\right\|\right] /(1-t) \\
& \leq \lambda \max \left\{\left\|x_{n}-p\right\|,\left\|x_{n}-f\left(x_{n}\right)\right\|\right\}
\end{aligned}
$$

For each $n$ such that the maximum is $\left\|x_{n}-p\right\|$, we have, using (12),

$$
\left\|x_{n+1}-p\right\| \leq\left(1-c_{n}\right)\left\|x_{n}-p\right\|^{2}+c_{n} \lambda^{2}\left\|x_{n}-p\right\|^{2}-c_{n}\left(1-c_{n}\right)\left\|f\left(x_{n}\right)-x_{n}\right\|^{2} .
$$

For each $n$ such that the maximum is $\left\|f\left(x_{n}\right)-x_{n}\right\|$, we have

$$
\left\|x_{n+1}-p\right\|^{2} \leq\left(1-c_{n}\right)\left\|x_{n}-p\right\|^{2}-c_{n}\left(1-c_{n}-\lambda^{2}\right)\left\|f\left(x_{n}\right)-x_{n}\right\|^{2} .
$$

In either case, we have

$$
\left\|x_{n+1}-p\right\|^{2} \leq\left\|x_{n}-p\right\|^{2}-c_{n}\left(1-c_{n}-\lambda^{2}\right)\left\|f\left(x_{n}\right)-x_{n}\right\|^{2} .
$$

The above inequality implies that $\left\{\left\|x_{n}-p\right\|\right\} \downarrow$ in $n$ for all $n$ sufficiently large. Also, since $\left\{c_{n}\right\}$ satisfies (iii) and $\overline{\lim }_{n} c_{n}<1-\lambda^{2}$, there exists a subsequence $\left\{x_{n_{k}}\right\}$ such that $\lim _{k}\left\|f\left(x_{n_{k}}\right)-x_{n_{k}}\right\|=0$.

$$
\begin{aligned}
\left\|f\left(x_{n_{k}}\right)-f\left(x_{n_{l}}\right)\right\| \leq & q\left\|x_{n_{k}}-x_{n_{l}}\right\|+r\left\|x_{n_{k}}-f\left(x_{n_{k}}\right)\right\| \\
& \left.+s\left\|x_{n_{l}}-f\left(x_{n_{l}}\right)\right\|+t\left\|x_{n_{k}}-f\left(x_{n_{l}}\right)\right\|+\left\|x_{n_{l}}-f\left(x_{n_{k}}\right)\right\|\right] . \\
\left\|x_{n_{k}}-x_{n_{l}}\right\| \leq & \left\|x_{n_{k}}-f\left(x_{n_{k}}\right)\right\|+\left\|f\left(x_{n_{k}}\right)-f\left(x_{n_{l}}\right)\right\|+\left\|f\left(x_{n_{l}}\right)-x_{n_{l}}\right\|, \\
\left\|x_{n_{k}}-f\left(x_{n_{l}}\right)\right\| \leq & \leq x_{n_{k}}-f\left(x_{n_{k}}\right)\|+\| f\left(x_{n_{k}}\right)-f\left(x_{n_{l}}\right) \|,
\end{aligned}
$$

and

$$
\left\|x_{n_{l}}-f\left(x_{n_{k}}\right)\right\| \leq\left\|x_{n_{l}}-f\left(x_{n_{l}}\right)\right\|+\left\|f\left(x_{n_{k}}\right)-f\left(x_{n_{l}}\right)\right\| .
$$

Therefore 


$$
\begin{aligned}
\left\|f\left(x_{n_{k}}\right)-f\left(x_{n_{l}}\right)\right\| \leq & {\left[(q+r+t)\left\|x_{n_{k}}-f\left(x_{n_{k}}\right)\right\|\right.} \\
& \left.\quad+(q+s+t)\left\|x_{n_{l}}-f\left(x_{n_{l}}\right)\right\|\right] /(1-q-2 t) \\
\leq & \lambda(1-\lambda)^{-1}\left[\left\|x_{n_{k}}-f\left(x_{n_{k}}\right)\right\|+\left\|x_{n_{l}}-f\left(x_{n_{l}}\right)\right\|\right],
\end{aligned}
$$

so that $\left\{f\left(x_{n_{k}}\right)\right\}$ is Cauchy, hence convergent. Call the limit $u_{\text {. Then }}$ $\lim _{k} f\left(x_{n_{k}}\right)=\lim _{k} x_{n_{k}}=u$.

$$
\|f(u)-u\| \leq\left\|f(u)-f\left(x_{n_{k}}\right)\right\|+\left\|f\left(x_{n_{k}}\right)-x_{n_{k}}\right\|+\left\|x_{n_{k}}-u\right\| .
$$

Since $f \in C$,

$$
\begin{aligned}
\left\|f(u)-f\left(x_{n_{k}}\right)\right\| \leq q\left\|u-x_{n_{k}}\right\| & +r\|u-f(u)\|+s\left\|x_{n_{k}}-f\left(x_{n_{k}}\right)\right\| \\
& +t\left[\left\|u-f\left(x_{n_{k}}\right)\right\|+\left\|x_{n_{k}}-f(u)\right\|\right] .
\end{aligned}
$$

Therefore

$$
\begin{aligned}
\|f(u)-u\| & \leq\left[(1+q+t)\left\|u-x_{n_{k}}\right\|+(1+s)\left\|f\left(x_{n_{k}}\right)-x_{n_{k}}\right\|+t\left\|u-f\left(x_{n_{k}}\right)\right\|\right] /(1-r) \\
& \leq\left[(1+\lambda)\left\|u-x_{n_{k}}\right\|+(1+\lambda)\left\|f\left(x_{n_{k}}\right)-x_{n_{k}}\right\|+\lambda\left\|u-f\left(x_{n_{k}}\right)\right\|\right] /(1-\lambda),
\end{aligned}
$$

and $f(u)=u$. Since $p$ is unique, $u=p \in E$. Combining $\lim _{k} x_{n_{k}}=p$ and $\left\{\left\|x_{n}-p\right\|\right\} \downarrow$ in $n$ for all $n$ large enough we get $\lim _{n} x_{n}=p$.

Theorem 8. Let $H$ be a Hilbert space, $f \in P_{2}, f: E \rightarrow E, E$ a compact, convex subset of $H$. Let $A$ be defined by (8) with $\left\{c_{n}\right\}$ satisfying (i)-(iii) $\overline{\lim }_{n} c_{n}=$ $c<1-k$. Then the iteration scbeme (1), (9) converges strongly to a fixed point of $f$.

Proof. From [20] $/$ has a fixed point in $E$. Call it $p$.

Since $f \in P_{2}$, there exists a number $k, 0 \leq k<1$, such that if $u, v \in E$, then $\|f(u)-f(v)\|^{2} \leq\|u-v\|^{2}+k\|(I-f) u-(I-f) v\|^{2}$. Thus, if $v=p$ and $u=x_{n}$, $\left\|f\left(x_{n}\right)-p\right\|^{2} \leq\left\|x_{n}-p\right\|^{2}+k\left\|x_{n}-f\left(x_{n}\right)\right\|^{2}$, and, substituting in (12), we obtain $\left\|x_{n+1}-p\right\|^{2} \leq\left\|x_{n}-p\right\|^{2}-c_{n}\left(1-c_{n}-k\right)\left\|f\left(x_{n}\right)-x_{n}\right\|^{2}$.

Since $c_{n}<1-k$ for all $n$ sufficiently large, and $\left\{c_{n}\right\}$ satisfies (iii), the above inequality yields $\left\{\left\|x_{n}-p\right\|\right\} \downarrow$ for all $n$ sufficiently large and $\lim _{k}\left\|f\left(x_{n_{k}}\right)-x_{n_{k}}\right\|=$ 0 for some subsequence $\left\{x_{n_{k}}\right\}$. Since $E$ is compact, there exists a subsequence of $\left\{x_{n_{k}}\right\}$, call it $\left\{x_{m}\right\}$ such that $\lim _{m} f\left(x_{m}\right)=\lim _{m} x_{m}=u$.

Using the triangular inequality and the fact that $f \in P_{2}$, 


$$
\begin{aligned}
\|f(u)-u\| & \leq\left\|f(u)-f\left(x_{m}\right)\right\|+\left\|\left(x_{m}-u\right)\right\| \\
& \leq\left[\left\|u-x_{m}\right\|^{2}+k\left\|(I-f) u-(I-f) x_{m}\right\|^{2}\right]^{1 / 2}+\left\|x_{m}-u\right\| \\
& \leq\left\|u-x_{m}\right\|+\sqrt{ } k\left(\|u-f(u)\|+\left\|x_{m}-f\left(x_{m}\right)\right\|\right)+\left\|x_{m}-u\right\| .
\end{aligned}
$$

Therefore $\|f(u)-u\| \leq\left[2\left\|u-x_{m}\right\|+\sqrt{ } k\left\|_{x_{m}}-f\left(x_{m}\right)\right\|\right] /(1-\sqrt{ } k)$, so that $f(u)=u$. The conditions $\lim _{m} x_{m}=u$ and $\left\{\left\|x_{n}-u\right\|\right\} \downarrow$ in $n$ for all $n$ sufficiently large yield $\lim _{n} x_{n}=u$.

Remarks. 1. The the orem of [10] is the special case of Theorem 8 with $c_{n}=(n+1)^{-1}$.

2. A pseudoc ontractive operator is one satisfying (14) with $k=1$. The orem 8 cannot be extended to pseudocontractive operators, because they need not have any fixed points. Even for those pseudocontractive operators with fixed points, the present method of proof cannot be used. The interested reader may consult [2] for a convergent iteration scheme for pseudocontractive operators $f$ satisfying a Lipschitz condition and $(f(x), x) \leq\|x\|^{2}$ for all $x$ in $S_{r}(0)$.

3. Specializing Theorem 8 to the reals, $E=[a, b]$, shows that condition (6) is not necessary for the iteration scheme (1)-(3) to converge to a fixed point for $f \in P_{2}$. A simple counterexample shows that the condition $c<1-k$ is necessary.

4. Theorem 8 shows that, for compact sets $E$, the conclusion of Theorem 12 of [2] can be strengthened to strong convergence, without the assumption that $U$ is demicompact. The iteration scheme used there is the special case of Theorem 8 with $c_{n} \equiv 1-\lambda, k<\lambda<1$.

5. Theorem 10 of [9] is the special case of Theorem 8 with $\lim _{n} c_{n}=0$.

Theorem 9. Let $H$ be a Hilbert space, $E$ a compact, convex subset of $H$, $f: E \rightarrow E, f \in Q$ with at least one fixed point $p \in E$, and $I-f$ closed. Let $A$ be defined by (8) with $\left\{c_{n}\right\}$ satisfying (i)-(iii) and $\overline{\lim }_{n} c_{n}=c<1$. Then the iteration scheme (1), (9) converges strongly to a fixed point of $f$.

Proof. Since $f \in Q,\left\|f\left(x_{n}\right)-p\right\|^{2} \leq\left\|x_{n}-p\right\|^{2}$. This inequality, along with (12), gives $\left\|x_{n+1}-p\right\|^{2} \leq\left\|x_{n}-p\right\|^{2}-c_{n}\left(1-c_{n}\right)\left\|f\left(x_{n}\right)-x_{n}\right\|^{2}$.

As in the proof of Theorem 8 , one obtains a subsequence, say $\left\{x_{m}\right\}$, of $\left\{x_{n}\right\}$ which converges. The result follows by appealing to The orem 3 , since conditions (iii) and $\overline{\lim }_{n} c_{n}=c<1$ imply (iv).

Remarks. 1. Theorem 3 of [16] and The orem 3 of [17] treat mappings $f \in P_{1}$ and subject to additional restrictions, while the compactness condition on $E$ is replaced by $E$ being closed and bounded.

2. The orem 8 of $[4]$ treats mappings $f \in Q$ under the weaker condition that $E$ is closed and convex but with additional hypotheses on $f$. 
3. Theorem 2 of [15] is a special case of The orem 9, since the complex plane is a Hilbert space.

4. For $E$ compact, Theorem 6 of [2] is the special case of Theorem 9 with $c_{n} \equiv \lambda$, for some $0<\lambda<1$.

The first of the following two theorems generalizes Theorem 2 of [5]. The proofs are omitted, since they are basically the same as those in [5].

Theorem 10. Let $X$ be a linear normed space, $f: X \rightarrow X, f \in Q$ with a fixed point $p \in X$. Let $A$ be any regular triangular matrix satisfying (4) and (5). Then for any $x_{0} \in X$, the iteration scheme (2), (3) generates bounded sequences $\left\{x_{n}\right\}$ and $\left\{\bar{x}_{n}\right\}$.

Theorem 11. Let $H$ be a Hilbert space, $f: H \rightarrow H, f \in P_{2}$ with a fixed point $p \in H$. Let $A$ be defined by (8) with $\left\{c_{n}\right\}$ satisfying (i)-(iii) and $\overline{\lim }_{n} c_{n}=c$, $c<1-k$. Then for any $x_{0} \in H$, the scheme (9) generates bounded sequences $\left\{x_{n}\right\}$ and $\left\{\bar{x}_{n}\right\}$.

Theorems 6-9 and 11-13 of [2] can all be extended to the class of iteration schemes defined by (1), (9) for any $\left\{c_{n}\right\}$ satisfying (i), (ii), and (iv). We simply state these generalizations, since their proofs are the same as their counterparts in [2] along with an occasional appeal to the theorem in [7]. In each of these theorems the underlying space is a Hilbert space.

Theorem 12 [2, Theorem 6]. Let $f \in P_{1}, f: E \rightarrow E, E$ bounded, closed, and convex. Suppose $f$ is demicompact. Then $F$, the set of fixed points of $f$ in $E$, is a nonempty convex set, and, for any $x_{0} \in E,\left\{c_{n}\right\}$ satisfying (i), (ii), and (iv), the iteration scheme (9) converges strongly to a fixed point of $f$ in $E$.

Theorem 13 [2, The orem 7]. Let $f \in P_{1}, f: E \rightarrow E, E$ closed, bounded, and convex, and / has exactly one fixed point $p \in E$. Then, for any $x_{0} \in E,\left\{c_{n}\right\}$ satisfying (i), (ii), and (iv), (9) converges weakly to $p$.

Theorem 14 [2, Theorem 8]. Let $f \in P_{1}, f: E \rightarrow E, E$ bounded, closed, and convex. Then, for any $x_{0} \in E$ and $\left\{c_{n}\right\}$ satisfying (i), (ii), and (iv), (9) converges weakly to $p$, and $p$ is a fixed point of $f$ in $E$.

Let $x \in H . R_{E} x$ is defined to be the closest point to $x$ in $E$.

Theorem 15 [2, Theorem 9]. Let $f \in P_{1}, f: E \rightarrow E, E$ closed, bounded, and convex. Suppose further that if $p \in \partial E$ and if $p=R_{E}(f(p))$, then $p$ is a fixed point of $f$.

Then $f$ has a fixed point in $E$ and, for any $x_{0} \in E,\left\{c_{n}\right\}$ satisfying (i), (ii), and (iv), the scheme 


$$
x_{n}=\left(1-c_{n}\right) x_{n-1}+c_{n} R_{E}\left(f\left(x_{n-1}\right)\right), \quad n \geq 1
$$

converges weakly to a fixed point of $f$ in $E$.

Theorem 16 [2, Theorem 11]. Suppose that $E$ is uniformly smootb and, under the bypotheses of Theorem 9 or 10 of [2], that $f$ is a demicompact mapping of $E$ into $H$. Then the iteration scheme (1), (13) converges strongly to a fixed point of $f$ in $E$.

Theorem 17 [2, Theorem 12]. Let $E$ be a bounded, closed, convex subset of $H, f: E \rightarrow E, f \in P_{2}$. Then for $x_{0} \in E,\left\{c_{n}\right\}$ satisfying (i), (ii), (iii) and $\overline{\lim }_{n} c_{n}=c<1-k$, the iteration scheme (9) converges weakly to a fixed point $p \in E$. If, in addition, $f$ is demicompact, then $\left\{x_{n}\right\}$ converges strongly to $p$.

Theorem 18 [2, Theorem 13]. Let $f \in P_{2}, f: B_{r}(0) \rightarrow H$ and satisfying the Leray-Schauder condition. Let $R: H \rightarrow B_{r}(0), R$ a retraction. Then, for any $x_{0} \in B_{r}(0)$, and any $\gamma$ sucb that $0<1-k<\gamma<1,\left\{c_{n}\right\}$ satisfying (i)-(iii) and $\overline{\lim }_{n} c_{n}=c<1-k$, the scheme

$$
x_{n}=\left(1-c_{n}\right) x_{n-1}+c_{n} R U_{\gamma} x_{n-1}, \quad U_{\gamma}=\gamma I+(1-\gamma) f
$$

converges weakly to a fixed point $p \in B_{r}(0)$. If, in addition, $f$ is demicompact, then $\left\{x_{n}\right\}$ converges strongly to $p$.

Comments. 1. Let $f$ be the shift operator, $E$ the unit ball in $H$. Let $A$ be defined by (8) with $c_{0}=1, c_{n}=1 / 2, n>0$. Let $e_{k}$ denote the $k$ th coordinate sequence. With $x_{0}=e_{0}$, from (9), $x_{n}=2^{-n} \Sigma_{k=0}^{n}\left(\begin{array}{l}n \\ k\end{array}\right) e_{k+1}$ for all $n>1$. Thus the sequence $\left\{x_{n}\right\}$ not only does not converge strongly, but it does not have any strongly convergent subsequences. On the other hand, every $g \in H^{\prime}$ is of the form $g(x)=(x, a)$ for some $a \in H$. Also, $g\left(x_{n}\right)=2^{-n} \Sigma_{k=0}^{n}\left(\begin{array}{c}n \\ k\end{array}\right) a_{k+1}$, so that $\lim _{n} g\left(x_{n}\right)=0$, and $\left\{x_{n}\right\}$ is weakly convergent. This example shows that compactness is a necessary condition in Theorems 8 and 9 , and that weak convergence is the best one can expect for a function in $P_{1}, Q$, or $P_{2}$ and $E$ merely closed, bounded, and convex.

2. It is a well-known result $\left[8, \mathrm{p}\right.$. 57] that if $\lim _{n} p_{n} / P_{n}=\lim _{n} c_{n}=c \neq 0$, then the corresponding summability method is equivalent to convergence. Thus Theorems 3 and 6, and the corresponding results quoted in [2] and [4] involve iteration schemes where the matrix involved is equivalent to convergence. Recalling the abundance of comparison theorems in summability theory, one is tempted to conjecture that most of the theorems of this paper are valid for any regular method satisfying (4) and (5) and strictly stronger than convergence. 
However, the interlacing of the iteration in (2) and (3) precludes the use of ordinary comparis on techniques. Consequently each summability method, or class of summability methods, must be investigated individually to determine if it will bring about convergence to a fixed point of $f$ when used in (1)-(3).

\section{REFERENCES}

1. H. G. Barone, Limit points of sequences and their transforms by methods of summability, Duke Math. J. 5 (1939), 740-752. MR 1, 218.

2. F. E. Browder and W. V. Petryshyn, Construction of fixed points of nonlinear mappings in Hilbert space, J. Math. Anal. Appl. 20 (1967), 197-228. MR 36 \#74.

3. L. B. Ciric, Generalized contractions and fixed-point theorems, Publ. Inst. Math. (Beograd) 12 (1971), 19-26.

4. W. G. Dotson, Jr., On the Mann iterative process, Trans. Amer. Math. Soc. 149 (1970), 65-73. MR 41 \#2477.

5. W. G. Dotson, Jr. and W. R. Mann, A generalized corollary of the Browder-Kirk fixed point theorem, Pacific J. Math. 26 (1968), 455-459. MR 38 \#5080.

6. R. L. Franks and R. P. Marzec, A theorem on mean value iterations, Proc. Amer. Math. Soc. 30 (1971), 324-326. MR 43 \#6375.

7. C. W. Groetsch, A note on segmenting Mann iterates, J. Math. Anal. Appl. 40 (1972), 369-372.

8. G. H. Hardy, Divergent series, Clarendon Press, Oxford, 1949. MR 11, 25.

9. B. P. Hillam, Fixed point iterations and infinite matrices, and subsequential limit points of fixed point sets, $\mathrm{Ph} . \mathrm{D}$. Dissertation, University of California, Riverside, Calif., June, 1973.

10. G. G. Johnson, Fixed points by mean value iteration, Proc. Amer. Math. Soc. 34 (1972), 193-194. MR 45 \#1006.

11. R. Kannan, Some results on fixed points. III, Fund. Math. 70 (1971), no. 2, 169177. MR 44 \#879.

12. - Construction of fixed points of a class of nonlinear mappings, J. Math. Anal. Appl. 41 (1973), 430-438.

13. W. R. Mann, Mean value methods in iteration, Proc. Amer. Math. Soc. 4 (1953), 506-510. MR 14, 988.

14. C. L. Outlaw, Mean value iteration of nonexpansive mappings in a Banach space, Pacific J. Math. 30 (1969), 747-750. MR 40 \#807.

15. C. L. Outlaw and C. W. Groetsch, Averaging iteration in a Banach space, Bull. Amer. Math. Soc. 75 (1969), 430-432. MR 39 \#835.

16. J. Reinermann, Über Toeplitzsche Iterationsverfahren und einige ihre Anwendungen in der konstruktiven Fixpunktheorie, Studia Math. 32 (1969), 209-227. [Zb. 176, 123]. MR $46 \# 2508$.

17. - Über Fixpunkte kontrahierender Abbildungen und schwach konvergente Toeplitz-Verfahren, Arch. Math. (Basel) 20 (1969), 59-64. MR 43 \#2583.

18. B. E. Rhoades, Fixed point iterations using infinite matrices, Preliminary report, Notices Amer. Math. Soc. 19 (1972), A-516. Abstract \#72T-B142. 
19. B. E. Rhoades, A comparison of various definitions of contractive mappings (in preparation).

20. J. Schauder, Der Fixpunktsatz in Functionräumen, Studia Math. 2 (1930), 171180.

21. T. Zamfirescu, Fix point theorems in metric spaces, Arch. Math. 23 (1972), 292298.

DEPARTMENT OF MATHEMATICS, INDIANA UNIVERSITY, BLOOMNGTON, INDIANA 47401 